\title{
Three-dimensional synchronous proton radiography for dynamic magnetic fields in laser-produced high- energy-density plasmas
}

\section{Zhonghai Zhao}

https://orcid.org/0000-0003-2051-1740

Shu-Kai He

Research Center of Laser Fusion

H. H. An

Shanghai Institute of Laser Plasma

Z. Lei

Peking University

Y. Xie

Peking University

W. Q. Yuan

Peking University

J. L. Jiao

Peking University

K. N. Zhou

Research Center of Laser Fusion, CAEP

J. J. Ye

Shanghai Institute of Laser Plasma

\section{Z. Y. Xie}

Shanghai Institute of Laser Plasma

\section{J. Xiong}

Shanghai Institute of Laser Plasma

\section{Z. H. Fang}

Shanghai Institute of Laser Plasma

\section{He}

Institute of Applied Physics and Computational Mathematics

W. Wang

Shanghai Institute of Laser Plasma

\section{Weimin Zhou}

Laser Fusion Research Center https://orcid.org/0000-0001-5474-7511

\section{Baohan Zhang}


Laser Fusion Research Center, China Academy of Engineering Physics

Shaoping Zhu

Institute of Applied Physics and Computational Mathematics

B. Qiao ( $\sim$ bqiao@pku.edu.cn )

Peking University

Article

Keywords: dynamic magnetic fields, 3D synchronous proton radiography, high-energy-density plasmas

Posted Date: November 17th, 2021

DOl: https://doi.org/10.21203/rs.3.rs-871300/v1

License: (9) This work is licensed under a Creative Commons Attribution 4.0 International License. Read Full License 


\title{
Three-dimensional synchronous proton radiography for dynamic magnetic fields in laser-produced high-energy-density plasmas
}

\author{
Z. H. Zhao ${ }^{\dagger},{ }^{1}$ S. K. He ${ }^{\dagger},{ }^{2}$ H. H. An ${ }^{\dagger},{ }^{3}$ Z. Lei, ${ }^{1}$ Y. Xie, ${ }^{1}$ W. Q. Yuan, ${ }^{1}$ J. L. Jiao, ${ }^{1}$ K. N. Zhou, ${ }^{2}$ J. J. Ye, ${ }^{3}$ \\ Z. Y. Xie, ${ }^{3}$ J. Xiong, ${ }^{3}$ Z. H. Fang, ${ }^{3}$ X. T. He, ${ }^{4}$ W. Wang, ${ }^{3}$ W. M. Zhou, ${ }^{2}$ B. H. Zhang, ${ }^{2}$ S. P. Zhu ${ }^{凶},{ }^{2,4}$ and B. \\ Qiao $^{\square 1}$ \\ 1) Center for Applied Physics and Technology, HEDPS, and SKLNPT, School of Physics, Peking University, \\ Beijing 100871, China \\ 2) Science and Technology on Plasma Physics Laboratory, Research Center of Laser Fusion, \\ China Academy of Engineering Physics (CAEP), Mianyang 621900, China \\ 3) Shanghai Institute of Laser Plasma, CAEP, Shanghai 201800, China \\ ${ }^{4)}$ Institute of Applied Physics and Computational Mathematics, Beijing 100094, \\ China
}

(Dated: 2 September 2021)

Understanding the generation and evolution of magnetic fields in high-energy-density plasmas is a major scientific challenge in broad research areas including astrophysics, cosmology, and laser fusion energy. However, the fully three-dimensional (3D) topologies of such dynamic magnetic fields are still unknown yet. Here we report experiments of the first $3 \mathrm{D}$ synchronous proton radiography for self-generated magnetic fields in respectively laser-produced low-Z $\mathrm{CH}$ and high- $\mathrm{Z} \mathrm{Cu}$ plasmas. The radiography images show that abundant $3 \mathrm{D}$ filamentary structures of magnetic fields grow up in coronal region of $\mathrm{CH}$ plasmas, while for $\mathrm{Cu}$, the fields are majorly compressed along the dense surface region whose internal structures are pretty vague. These results are reproduced and explained by a combination of radiation-magnetohydrodynamic, particle-in-cell and Vlasov-Fokker-Planck simulations, where the cross-scale effects of Biermann battery, Nernst advection, resistive diffusion, Righi-Leduc and particularly kinetic Weibel instability are all taken into account. Our findings provide much enlightenment to the role of magnetic field generation in implosion and hohlraum dynamics of laser fusion.

$\mathrm{M}$ AGNETIC fields are ubiquitous in the universe, permeating across all scales of matters from astrophysical objects to laboratories ${ }^{1-4}$. This is not a surprise since almost all normal (nondark) matter that makes up our cosmos exist in form of plasmas, in many cases, high-energy-density (HED) plasmas ${ }^{5}$ with energy densities above $10^{5} \mathrm{~J} / \mathrm{cm}^{3}$. Magnetic fields play important roles in many topics of astrophysics such as jets, star and planet formation, accretion discs, compact star mergers, supernovae bursts, to name just a few. In laboratory fusion energy studies, specifically inertial confinement fusion (ICF), that is hoped to make clean fusion power a reality on Earth by high-power lasers, either asymmetries in ablation of the low- $\mathrm{Z}$ capsule or nonlinear interactions between the high- $\mathrm{Z}$ hohlraum wall and incident lasers results in generation of magnetic fields, which significantly impact the implosion dynamics of directdrive scheme and hohlraum dynamics of indirect-drive scheme.

In HED plasmas, according to the generalized form of the Ohm's law ${ }^{6}$, at least 13 terms contribute to the generation and evolution of magnetic fields as

$$
\frac{\partial \mathbf{B}}{\partial t}=\frac{\nabla T_{e} \times \nabla n_{e}}{e n_{e}}+\nabla \times\left[\left(\mathbf{u}+\mathbf{v}_{N}\right) \times \mathbf{B}\right]-\nabla \times\left(\frac{\eta}{\mu_{0}} \nabla \times \mathbf{B}\right)-\nabla \times\left(\frac{\mathbf{J} \times \mathbf{B}}{e n_{e}}\right)+\cdots,
$$

where the first term at the right hand side is the so-called Biermann Battery source ${ }^{7}$, implying that strong magnetic fields can be self-generated by the non-collinear electron temperature and density gradients $\nabla T_{e} \times \nabla n_{e}$; the second term represents the magnetic convection by the plasma flow velocity $\mathbf{u}$ and the so-called Nernst velocity $\mathbf{v}_{N}=$ $\left\langle\mathbf{v} v^{3}\right\rangle_{f 1} / 2\left\langle v^{3}\right\rangle_{f 0}$ arising from the heat-carrying electrons ${ }^{8}$; the third term is the magnetic diffusion caused by the electric resistivity $\eta$; and the physics of other terms can be found in the derivations of Hains ${ }^{6}$. As a result, the dynamics of self-generated magnetic fields in HED plasmas are rather complicated and unclear yet so far.

One of the most critical issue for the magnetic field dynamics is that they are intrinsically connected to that of electron heat transport, which in HED plasmas is commonly nonlocal ${ }^{9,10}$, as the linearized Spitzer Härm theory breaks down due to the long mean free path of hot electrons compared to the temperature gradient scale length. Such an intricate connection between magnetic field and heat flow is manifested in a strongly coupled way, where the classical Braginskii model ${ }^{11}$ becomes invalid. On the one hand, the nonlocal heat flow $Q_{N L}=\left\langle\frac{1}{2} m_{e} v^{2} \mathbf{v}\right\rangle_{f 1}$ modifies the above

$\dagger$ These authors have contributed to this work equally.

$\bowtie$ Corresponding author. e-mail: bqiao@pku.edu.cn, zhu_shaoping@iapcm.ac.cn. 


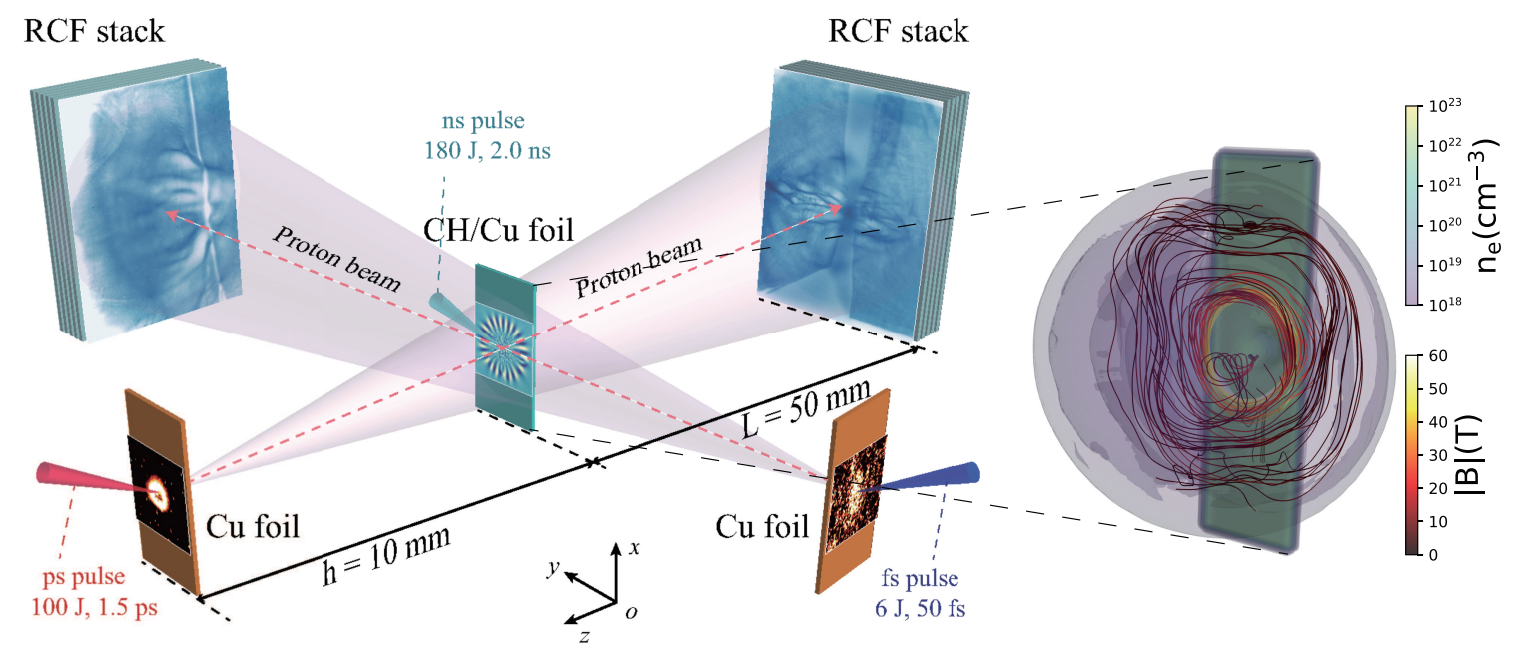

Fig 1. Experimental setup. The materials of the main foil targets (green) are respectively plastic $\mathrm{CH}_{(}\left(\mathrm{C}_{1} \mathrm{H}_{1}\right)$ with density $\rho=1.02 \mathrm{~g} / \mathrm{cm}^{3}$ and $\mathrm{Cu}$ with $\rho=8.92 \mathrm{~g} / \mathrm{cm}^{3}$. Both foil targets have thickness of $30 \mu \mathrm{m}$ and narrow area of $3 \mathrm{~mm} \times 0.8 \mathrm{~mm}$. The HED plasmas are produced from the main foil targets by ablation with a $2 \omega$ ns laser pulse (green) that have energy of $180 \mathrm{~J}$ in a $2 \pm 0.1 \mathrm{~ns}$ square temporal profile and $160-\mu \mathrm{m}$-diameter Gaussian focal spot. The average intensity of the ns pulse is $5.0 \times 10^{14} \mathrm{~W} / \mathrm{cm}^{2}$. The $3 \mathrm{D}$ proton radiography are set up along both the face-on (-z-axis) and side-on ( $y$-axis) directions. Two ultrafast radiography proton beams are generated at almost the same time from respectively the backlighter 15 - $\mu$ m-thick $\mathrm{Cu}$ foil (yellow) irradiated by a ps pulse (red) and $7-\mu$ m-thick $\mathrm{Cu}$ foil (yellow) by a fs pulse (purple). The ps pulse has energy $100 \mathrm{~J}$, wavelength $1.053 \mu \mathrm{m}$, duration $1.5 \mathrm{ps}$ and is focused with a $800 \mathrm{~mm}$ focal-length, $f / 2.5$ off-axis parabolic mirror to an intensity about $1.0 \times 10^{19} \mathrm{~W} / \mathrm{cm}^{2}$. And the fs pulse has energy $6 \mathrm{~J}$, wavelength $800 \mathrm{~nm}$, duration $50 \mathrm{fs}$ and is focused to an intensity about $5.0 \times 10^{19} \mathrm{~W} / \mathrm{cm}^{2}$. The deflected protons are finally deposited on the HD-V2 radiochromic film (RCF) stacks, whose response to proton dose has been well calibrated with a good linear relationship between proton dose and optical density (OD). The distances between the backlighter foils (yellow) and the main foil (green) are both $h \sim 10 \mathrm{~mm}$, and those between the main foil and the RCF stacks are about $L \sim 50 \mathrm{~mm}$, so the geometric magnification factors of the proton radiography system in both directions are about $M \equiv(h+L) / h \approx 6.0$. 3D distributions of plasma density $\left(n_{e}\right)$ and self-generated magnetic field $(|B|)$ from RMHD simulations are also shown at the right side, where the laser-irradiated $\mathrm{CH}$ target is chosen.

Nernst velocity $\mathbf{v}_{N}{ }^{9}$, which results in convection and compression of magnetic fields down the temperature gradient, and hence, influences their evolutions. In turn, the fields act back on the heat flow transport via affecting electron motions. On the other hand, through the Righi-Leduc heat flow ${ }^{12}$, the magnetic fields affect electron motions in the direction orthogonal to both themselves and the temperature gradient, causing significant bending of the heat flux and also influencing field evolutions. Furthermore, more complicatedly, magnetic field evolutions in HED plasmas are also determined by a series of hydrodynamic (such as Rayleigh-Taylor ${ }^{13,14}$ ) and kinetic (Weibel ${ }^{15-18}$ ) instabilities as well as reconnections ${ }^{19-23}$ across various temporal and spatial scales. Therefore, although a large amount of efforts have been made, profound questions remain over the generation, evolution and role of magnetic fields in HED plasmas, which are essentially three-dimensional (3D) and cross-scale problems. Severe technical challenges exist for both experimental and numerical studies.

From the experimental perspective, to give a full picture of the above coupled dynamics, highly-resolved 3D topologies of magnetic field evolutions need to be measured. Proton radiography ${ }^{24,25}$ is an important magnetic-field diagnostic used in HED experiments, with applications spanning laboratory astrophysics and ICF. The diagnostic is implemented by passing an approximately uniform beam of imaging protons through HED plasmas onto a spatially resolved detector. While inside the plasma, the protons experience deflection by the Lorentz forces of magnetic fields, resulting in a redistribution of image-flux. However, so far, no 3D synchronous proton radiography experiments have been carried out yet, which technically requires synchronization of multiple laser-driven proton beams from both faceon and side-on directions. From the numerical modeling point of view, the holy grail is comprehensive understanding of the coupling between the micro and macro scale processes in magnetized HED plasmas that typically have a very large degree of freedom at all scales, therefore, a cross-scale, comparatively self-consistent combination of RMHD and kinetic [particle-in-cell (PIC) and/or Vlasov-Fokker-Planck (VFP)] simulations is required.

To resolve the above issues, here we report the first 3D synchronous proton radiography experiments for selfgenerated magnetic fields in respectively laser-produced low-Z $\mathrm{CH}$ and high-Z $\mathrm{Cu}$ plasmas, where the unique synchronization technology of three laser pulses [femtosecond (fs), picosecond (ps) and nanosecond (ns) pulses] are applied. 
The two plasma materials can be representative of the HED plasmas from respectively laser-ablated capsule in directdrive ICF scheme and laser-irradiated hohlraum wall in indirect-drive scheme. A series of spatially and temporally highly-resolved radiographys help to understand the importance of various physical effects that influence magnetic field dynamics in HED plasmas. The experimental results are supported by a combination of cross-scale RMHD, PIC and VFP simulations.

\section{RESULTS}

\section{Experimental setup}

The first 3D synchronous proton radiography experiments are carried out on the unique XingGuang-III (XG-III) laser facility, which has three orthogonal pulses (the fs, ps and ns pulses) synchronized with each other with almost no jitter time ${ }^{26}$, see Methods. Figure 1 shows a diagram of the experimental setup, where, for convenience of discussion, a right-handed Cartesian coordinate system $o-x y z$ is established. The HED plasmas are produced from the $\mathrm{CH} / \mathrm{Cu}$ foil target by ablation with a ns laser pulse. The 3D proton radiography are set up along both face-on $(-z$-axis $)$ and side-on ( $y$-axis) directions, where two ultrafast radiography proton beams are generated synchronously by target normal sheath accelerations using respectively ps and fs pulses. The jitter between the fs and ps pulses is less than 1.32 ps. Both proton beams are of high qualities with almost uniform distributions, where the static radiography images are shown in Supplementary Fig. S1. The uncertainty for the proton propagation distance is $\delta h \sim \pm 0.3 \mathrm{~mm}$, corresponding to typical proton energy $E_{p} \approx 3.9 \mathrm{MeV}$, which indicates time uncertainty of $\delta t \sim \pm 10 \mathrm{ps}$. This is much less than the characteristic time of plasma evolution, so that 3D synchronous proton radiographys are guaranteed.

\section{D radiographys for self-generated magnetic field evolutions}

The experimental results are shown in Fig. 2, which include both face-on and side-on radiographys for respectively laser-produced $\mathrm{Cu}[\mathbf{2}(\mathrm{a})-\mathbf{2}(\mathrm{e})]$ and $\mathrm{CH}[\mathbf{2}(\mathrm{f})-\mathbf{2}(\mathrm{j})]$ plasmas at times $t=1.8,2.4$ and $3.0 \mathrm{~ns}$ from laser irradiations. First, let's see their overall outlines. At $t=1.8 \mathrm{~ns}$ when laser is still on, we see from the side-on radiographys 2(a) and 2 (f) that for both $\mathrm{Cu}$ and $\mathrm{CH}$ plasmas protons are deflected towards the outside region of the expanding plasma bubbles, while from the face-on $\mathbf{2}(\mathrm{d})$ and $\mathbf{2}$ (i) that protons are deflected towards the inner side (blue curves). From such characters of $3 \mathrm{D}$ radiographys, we conclude that the main contributions of proton deflections in both cases are the toroidal magnetic fields $B_{\theta}$ rather than the monopole electric fields ${ }^{23}$, in consistence with the expected Biermann battery effect $\nabla T_{e} \times \nabla n_{e}$, see also 3D RMHD simulation results in Fig. 1. From these 3D outlines, we also estimate the plasma expansion velocities in different directions. For $\mathrm{CH}$ plasmas, we get from the side-on 2 (f) that the mean expansion velocities as $v_{x} \sim 700 \mathrm{~km} / \mathrm{s}$ and $v_{z} \sim 1000 \mathrm{~km} / \mathrm{s}$, and from face-on $2(\mathrm{i})$ that $v_{y} \sim 500 \mathrm{~km} / \mathrm{s}$. Such differences of plasma expansion velocities in three directions just imply that the plasma properties (temperature, density etc.) are asymmetric, which is the prerequisite condition for generation of $B_{\theta}$ [see the blue circle in 2(i)]. Further, due to the similar charge to mass ratios after ionization, $\mathrm{Cu}$ plasmas have the similar expansion velocities as $\mathrm{CH}$.

Secondly, from spatially and temporally highly-resolved Fig. 2, we also see that the self-generated magnetic fields in $\mathrm{Cu}$ and $\mathrm{CH}$ plasmas have different internal structures. Abundant 3D filamentary field structures develop in $\mathrm{CH}$ hot coronal plasmas [see 2(f)], while in Cu plasmas, the fields' internal structures are pretty vague [2(a)]. At later times $t=2.4$ and $3.0 \mathrm{~ns}$ after the laser is over, the filamentary structures in $\mathrm{CH}$ plasmas $[\mathbf{2}(\mathrm{g})$ and $\mathbf{2}(\mathrm{h})]$ even grow up more strongly for a long time, while in $\mathrm{Cu}$ plasmas, the fields are gradually compressed and concentrated on the cold dense surface region [see the red circle region in $\mathbf{2}(\mathrm{b})$ and $\mathbf{2}(\mathrm{c})$ ] whose internal structures disappear. Furthermore, from the combination of side-on $[\mathbf{2}(\mathrm{g})]$ and face-on $[\mathbf{2}(\mathrm{j})]$ radiographs, we conclude that the filamentary structure are indeed 3D. Another worth mentioned is that the sheath electric field along the $\mathrm{Cu}$ foil surface [marked by the blue thick line in $2(\mathrm{a})]$ is $<10^{7} \mathrm{~V} / \mathrm{m}$, much weaker than that for $\mathrm{CH}$ target $\left(3 \times 10^{8} \mathrm{~V} / \mathrm{m}\right)[\mathbf{2}(\mathrm{f})]^{27}$, and both of them disappear at later time when laser is over due to the return current effect.

To extract more quantitative information from the radiographys, we use the inverse field-reconstruction code "PROBLEM" 28 to recover the path-integrated magnetic fields $\psi$, see Methods. Reconstructed $\psi$ for the red-box regions in Figs. 2(f)-2(h) and 2(j) are shown in $2(\mathrm{k})-\mathbf{2}(\mathrm{m})$ and $\mathbf{2}(\mathrm{o})$ respectively, which further confirms the existences of stochastic filamentary fields in $\mathrm{CH}$ plasmas. The local characteristic spatial scales $\lambda$ [for the black dashed regions in $\mathbf{2}(\mathrm{k})-\mathbf{2}(\mathrm{m})]$ and the average scale $\bar{\lambda}=2 \pi\left[\int_{k_{\min }}^{k_{\max }} E_{B}(k) / k d k\right] /\left[\int_{k_{\min }}^{k_{\max }} E_{B}(k) d k\right]$ of the filaments are shown in Fig. 2(n), where $E_{B}(k)$ is the Fourier energy spectrum, $k_{\min }$ and $k_{\max }$ are the lower and upper bounds of wave vectors. Both characteristic scales of filaments are around several $100 \mu \mathrm{m}$ and increase with time, from which we infer that the typical strengths of the filamentary fields are about a few up to $10 \mathrm{~T}$, remaining even long after laser is over. 

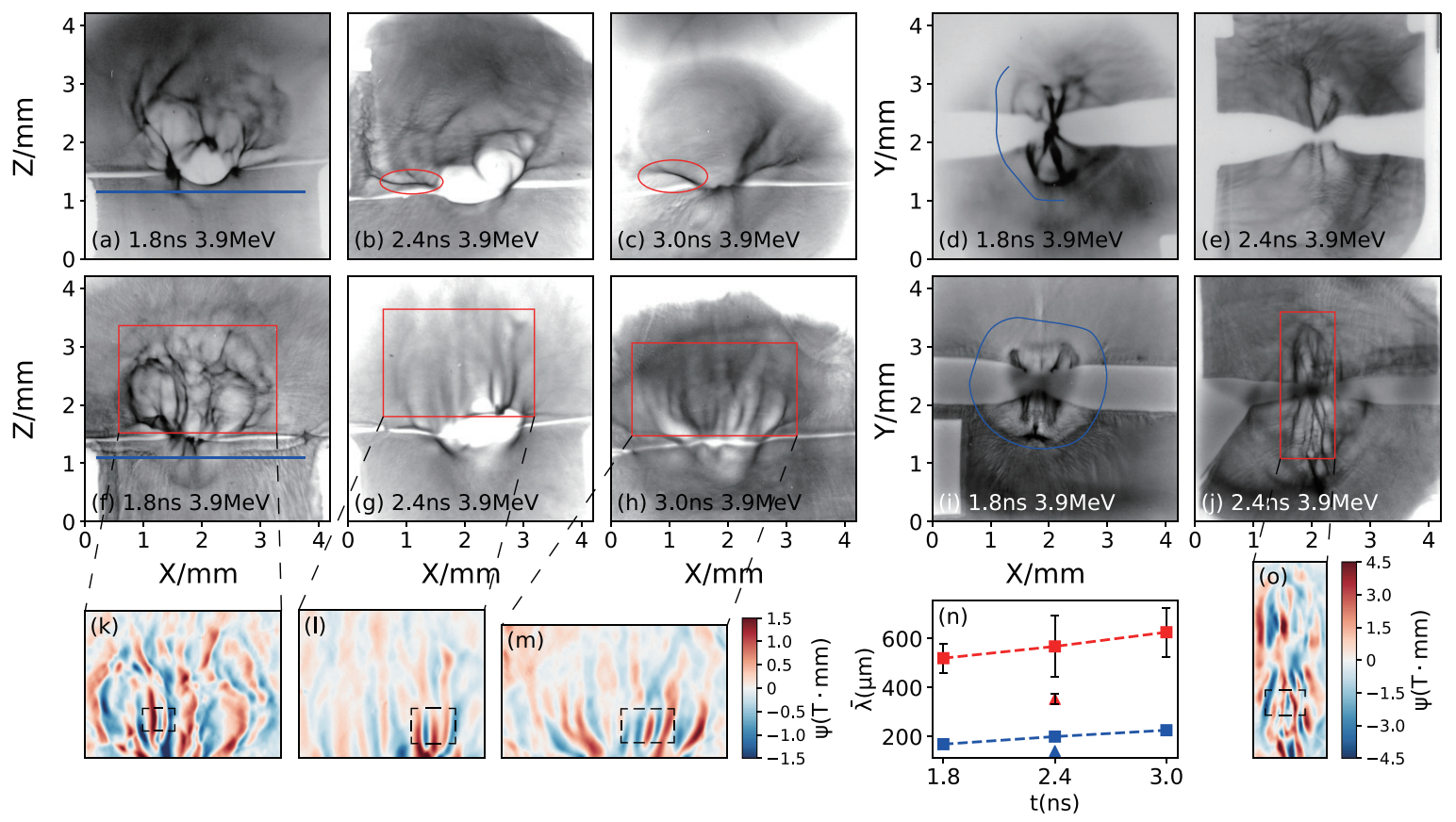

Fig 2. 3D synchronous proton radiographys for self-generated magnetic fields in $\mathbf{H E D} \mathbf{C u}$ and $\mathrm{CH}$ plasmas. The upper row shows radiographys for $\mathrm{Cu}$ plasmas of respectively side-on [(a)-(c)] and face-on [(d) and (e)] directions; the middle row shows those for $\mathrm{CH}$ of respectively side-on [(f)-(h)] and face-on [(i) and (j)] at times $t=1.8,2.4$ and 3.0 ns, where the higher the grayscale represents the higher the proton doses, the coordinates of $\mathrm{X}, \mathrm{Y}$ and $\mathrm{Z}$ axes are at the realist spatial scales of HED plasmas after transforming from the RCF images by dividing the magnification factors $M=6.0$, and the detailed time and radiography proton energy are labeled for each subfigures. The path-integrated magnetic fields $\psi$ (in units of $\mathrm{T} \cdot \mathrm{mm}$ ) after inverse field reconstruction of the filamentary structures in the red-box regions of (f)-(h) and (j) are shown in respectively $(\mathrm{k})-(\mathrm{m})$ and $(\mathrm{o})$. The local characteristic spatial scale $\lambda$ [the black dashed box region in (k)-(m) and (o)] (in blue) and the average spatial scale (in red) of these filamentary magnetic fields varying with time are shown in (n), where the square and triangle symbols correspond to those of respectively side-on and face-on directions, and the error bars are calculated by one standard deviation.

Furthermore, comparing $\mathbf{2}(\mathrm{l})$ and $\mathbf{2}(\mathrm{o})$ we see that $\psi$ obtained from face-on radiography is stronger than that from side-on, while its characteristic scale is smaller than the latter [2(n)]. This again verifies $3 \mathrm{D}$ asymmetric distributions of magnetic fields.

\section{Combined RMHD and PIC simulations for filamentary magnetic field structures in underdense corona plasmas}

To explain the experimentally-observed complex structures of magnetic fields in HED plasmas, a comprehensive understanding of the coupling between the micro- and macro-scale dynamics of HED plasmas is required. For this, we use the cross-scale combination of RMHD, PIC and VFP simulations. First, let's concentrate on the filamentary structures in underdense corona plasma region. The expanding HED plasma parameters after ns laser ablations are obtained by RMHD simulations using the code "FLASH" ${ }^{29}$, which has been extended with the self-consistent magnetic field modeling capabilities (details see Methods). The initial laser and target conditions of RMHD simulations are set following those in experiments, also see Methods for details. The obtained $\mathrm{CH} / \mathrm{Cu}$ plasma parameters including densities, temperatures and ion charge states are shown in Supplementary Figs. S2 and S3. These parameters are then employed and scaled down as a reference for setup of the initial conditions for the following 2D collisional PIC simulations with the code "EPOCH" 30 (see Methods). Furthermore, to keep the consistency between PIC and RMHD simulations, two key characteristic times need to be considered: the characteristic time for formation of the temperature anisotropy $t_{a n i}=l_{T} / v_{T e}{ }^{31}$, where $l_{T}$ and $v_{T e}$ are the temperature gradient length and electron thermal velocity respectively, and the characteristic collisional time $t_{\text {coll }}=1 / \nu_{e}$, where $\nu_{e} \propto(1+\bar{Z}) n_{e} / T_{e}^{3 / 2}$ is the total electron collision rate. Since the collisions tend to undermine the anisotropy, we define a key dimensionless parameter $\alpha=t_{\text {ani }} / t_{\text {coll }}=\nu_{e} l_{T} / v_{T e}$ as a correlation of two characteristic times. As shown in Fig. 3(g), to keep the PIC simulation system as similar as possible to RMHD system, we adjust $\nu_{e}$ of $\mathrm{Cu}$ and $\mathrm{CH}$ plasmas in PIC simulations so that their $\alpha$ (cross and star symbols) match well with those (dashed and solid lines) in RMHD, and $\alpha$ is about 20 

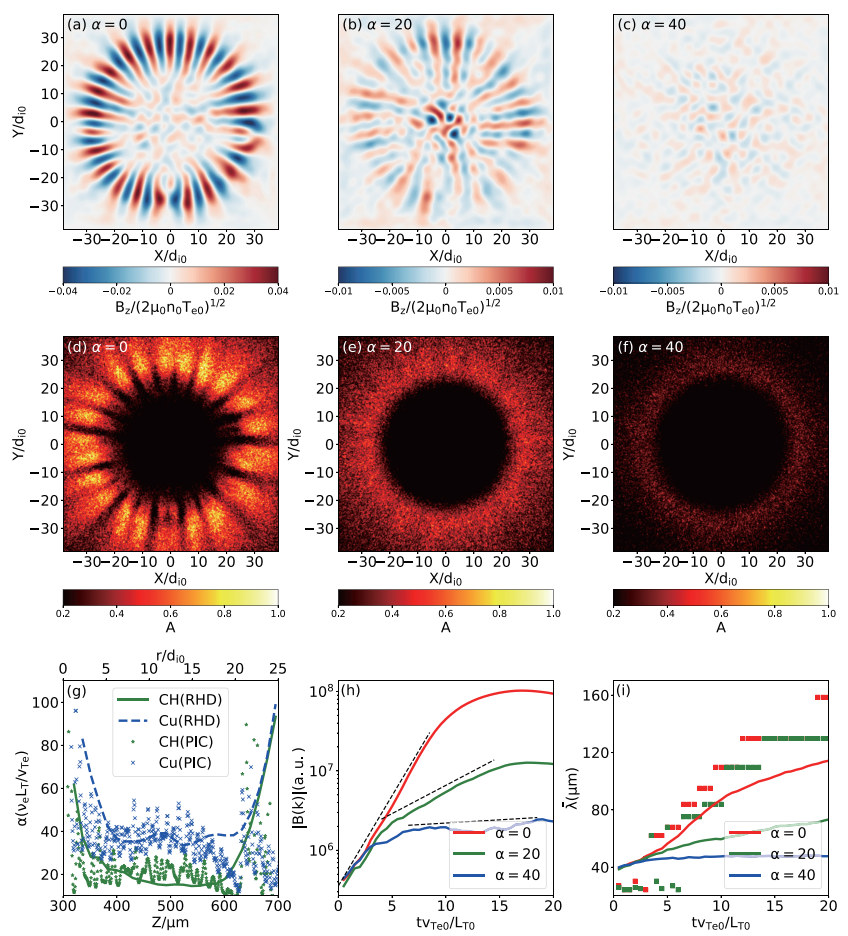

Fig 3. Combined RMHD and PIC simulation results for filamentary magnetic field structures. (a)-(c) Strength maps for out-of-plane magnetic field $B_{z}$ for respectively collisionless $(\alpha=0)$, $\mathrm{CH}(\alpha=20)$ and $\mathrm{Cu}(\alpha=40)$ plasmas at time $t=15 L_{0} / v_{t e 0}$. (d)-(f) The corresponding temperature anisotropy parameters $\mathcal{A}$. (g) The key parameters $\alpha=\nu_{e} l_{T} / v_{T e}$ for respectively $\mathrm{CH}$ (solid line) and $\mathrm{Cu}$ (dashed line) plasmas in RMHD simulations at $t=1.6 \mathrm{~ns}$, in comparison with those (star symbols for $\mathrm{CH}$ and crosses for $\mathrm{Cu}$ ) in PIC simulations at $t=15 L_{0} / v_{t e 0}$. (h) Time evolutions of $\left|B_{z}(k)\right|$ for the most unstable mode of the Weibel instabilities in three cases. (i) The local characteristic spatial scales $\lambda$ (scattered points) and the average spatial scale $\bar{\lambda}$ (solid lines) of the filaments, where the position is chosen to be with local plasma densities of $n_{e, l o c} \sim 10^{19} \mathrm{~cm}^{-3}$.

and 40 for $\mathrm{CH}$ and $\mathrm{Cu}$ plasmas, respectively.

Figures 3(a)-3(c) show maps of out-of-plane magnetic fields $B_{z}$ for respectively collisionless $(\alpha=0), \mathrm{CH}(\alpha=20)$ and $\mathrm{Cu}(\alpha=40)$ plasmas at $t=15 L_{0} / v_{t e 0}$ in PIC simulations. The corresponding anisotropy parameters $\mathcal{A} \equiv$ $2 T_{a n i} /\left(T_{i s o}-T_{a n i}\right)^{32,33}$ are also displayed in $\mathbf{3}(\mathrm{d})-\mathbf{3}(\mathrm{f})$, where $T_{i s o}=\left(T_{x x}+T_{y y}\right) / 2$ and $T_{a n i}=\left(\left(T_{x x}-T_{y y}\right)^{2}+4 T_{x y}^{2}\right)^{1 / 2} / 2$ represent the isotropic and anisotropic components, respectively, and $T_{i j}(i$ and $\mathrm{j}=\mathrm{x}, \mathrm{y})$ is the electron temperature tensor. We see that abundant filaments of magnetic fields significantly develop in collisionless or weak collisional $\mathrm{CH}$ plasmas, where, correspondingly, the plasma temperatures show pronounced anisotropic distributions. This makes us conclude that the filamentary structures of magnetic fields in $\mathrm{CH}$ corona plasmas originate from the thermal-driven Weibel instabilities ${ }^{34}$, because, as we know, the Weibel instability is just induced by the temperature anisotropy $\mathcal{A}^{15,16}$. Under experimental conditions $n_{e 0} \sim 10^{20} \mathrm{~cm}^{-3}$ and $T_{e 0} \sim 1500 \mathrm{eV}$ (see Supplementary), we estimate the filamentary field strength in 3(b) as $B_{z, \mathrm{CH}} \sim 0.01 \sqrt{2 \mu_{0} n_{0} T_{e 0}} \sim 2.5 \mathrm{~T}$, in consistence with the above estimations from experiments $[\mathbf{2}(\mathrm{g})$ and $\mathbf{2}(\mathrm{h})]$. However, for collisional $\mathrm{Cu}$ plasmas $[\mathbf{3}(\mathrm{c})]$, we see only weak random fluctuations exist with almost no filamentary structures, also in agreement with the above experiments [2(b) and 2(c)]. This is because the collisions in $\mathrm{Cu}$ lead to heavy suppression of the Weibel instabilities ${ }^{31,35}$. Note that the collision rate $\nu_{e}$ increases with plasma density and $l_{T}$ also increases when the temperature gradient decreases, both resulting in increase of $\alpha$ (see Supplementary Figs. S3 and S4), therefore, the Weibel instability with filaments occurs only in the hot underdense corona plasma region.

Time evolutions of $\left|B_{z}(k)\right|$ for the most unstable mode of the Weibel instabilities in three cases are shown in Fig. 3(h). All of them exhibit approximately exponential growths with time and the growth rate in $\mathrm{Cu}$ plasmas is indeed heavily suppressed due to the collision effect. The corresponding e-folding times of the most unstable mode for three cases are estimated as respectively $t_{1} \sim 1.91 L_{0} / v_{T e 0}(\alpha=0), t_{2} \sim 6.11 L_{0} / v_{T e 0}(\alpha=20)$ and $t_{3} \sim 53.5 L_{0} / v_{T e 0}$ $(\alpha=40)$. Considering the variation of plasma density profiles during long-time evolutions, the local plasma scale lengths are $L_{l o c} / v_{T e, l o c} \sim 10 L_{0} / v_{T e 0}$, see Supplementary Fig. S5 and the corresponding discussions. Since PIC and RMHD simulations are self-similar, we obtain from RMHD simulations that $L_{l o c} \sim 500 \mu \mathrm{m}$ and $v_{T e, l o c}=10^{7} \mathrm{~m} / \mathrm{s}$ $\left(T_{e, l o c} \sim 600 \mathrm{eV}\right)$. Substituting these parameters into the e-folding time relations that we get from PIC simulations, we obtain that in experiments the e-folding time for the Weibel instability in $\mathrm{CH}$ plasmas is about $t_{\mathrm{CH}, \mathrm{WI}} \sim 30 \mathrm{ps}$ 


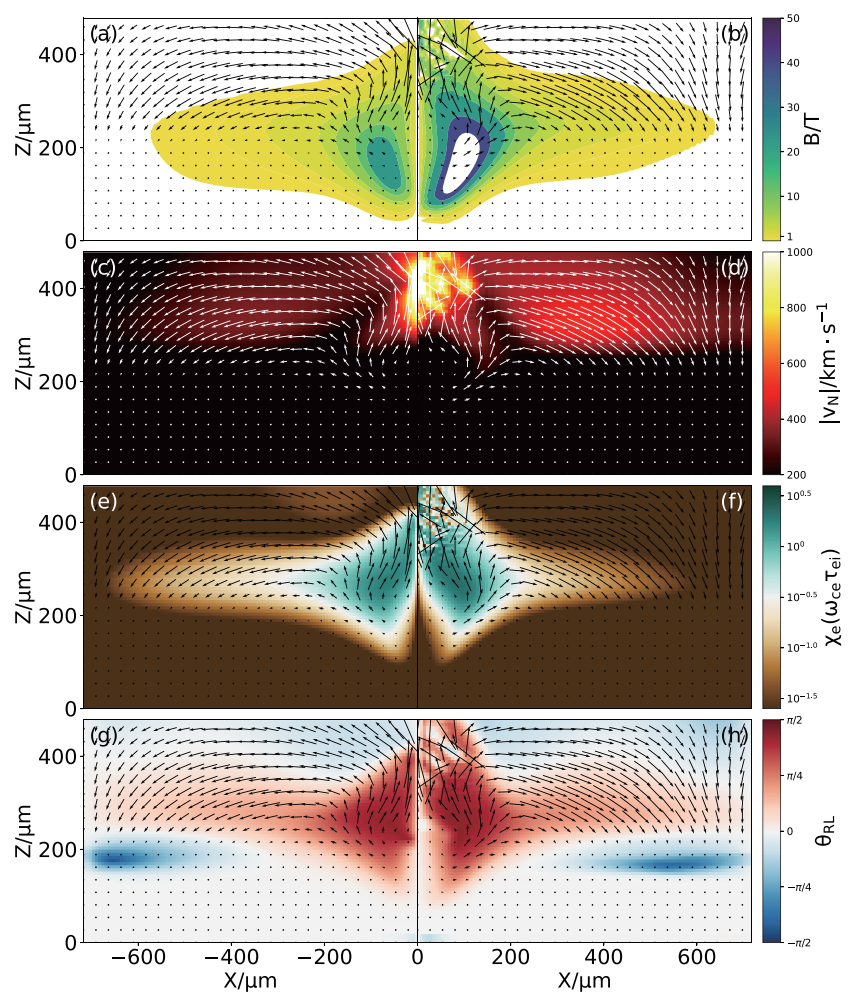

Fig 4. Combined RMHD and VFP simulation results for magnetic field transport in overdense plasmas. Distribution maps of the self-generated magnetic field $B\left[(\mathrm{a})\right.$ and (b)], Nernst velocity $\left|v_{N}\right|[(\mathrm{c})$ and (d)], magnetization (hall) parameter $\chi_{e}\left[(\mathrm{e})\right.$ and (f)], and Righi-Leduc angle $\theta_{R L}[(\mathrm{~g})$ and $(\mathrm{h})]$ in respectively $\mathrm{CH}$ (left column) and Cu (right column) plasmas at $t=300 \mathrm{ps}$. The arrows in all subfigures represent distributions of the Nernst velocity field $\mathbf{v}_{N}$, whose directions and lengths correspond to respectively the directions and relative magnitudes of $\mathbf{v}_{N}$.

and that in $\mathrm{Cu}$ plasmas is $t_{\mathrm{Cu}, \mathrm{WI}} \sim 260 \mathrm{ps}$. Generally, the Weibel instability growth time is about several times of the e-folding time, that is, sufficient development of the Weibel instability in $\mathrm{CH}$ plasmas needs about 100 ps and that in $\mathrm{Cu}$ plasmas needs about much longer as $1 \mathrm{~ns}$. This further explains why the filamentary structures of magnetic fields in $\mathrm{CH}$ plasmas are much more pronounced than those in $\mathrm{Cu}$ plasmas in experiments. The local characteristic scale $\lambda$ (scattered points) and the average spatial scale $\bar{\lambda}$ (solid lines) of the filaments in PIC simulations are also displayed in Fig. 3(i). We see that all of them increase with time and reach the order up to 100s of $\mu \mathrm{m}$, in the same order of the experimental results $[\mathbf{2}(\mathrm{n})]$. It is worth noting that the Weibel instability keeps growing even after laser is over, and when it enters into the nonlinear and saturation stage, the nonlinear current coalscence further increase the scale of filaments ${ }^{16,36}$. This explains our experimental observation in Fig. 2(h) where the filaments keep growing at much later time after laser is over.

\section{Combined RMHD and VFP simulations for magnetic field transport in overdense plasmas}

In the overdense plasma region where laser cannot arrive, the heat energy is transported by hot electrons. Therefore, the transport of self-generated magnetic fields is strongly coupled with that of the electron heat flux, where the Nernst and Righi-Leduc effects play important roles. To model the coupled field-heat transport, kinetic VFP simulations with the code "OSHUN" 37 are carried out. The setup and initial conditions of simulations are shown in Methods and Supplementary Fig. S6. Figures 4(a) and 4(b) show the obtained distribution maps of magnetic fields in respectively $\mathrm{CH}$ and $\mathrm{Cu}$ overdense plasmas. We see that, for both cases, the peaks of magnetic fields locate near the laser focal spot, and the farther away, the weaker the field, which are consistent with the experiment results in Fig. 2 and RMHD simulations in Fig. 1. Due to the stronger inverse bremsstrahlung absorption at near-critical density region, $\mathrm{Cu}$ plasmas have higher temperature $T_{e}$ and steeper density gradient, resulting in much stronger magnetic field generation than in $\mathrm{CH}$ plasmas. The arrow symbols in 4(a) and 4(b) represent the Nernst convection velocity field $\mathbf{v}_{N}$, showing that the Nernst convection makes magnetic fields transport away from the focal spot towards the cold dense region. We also see from $4(\mathrm{c})$ and $4(\mathrm{~d})$ that $\left|v_{N}\right| \sim 600 \mathrm{~km} / \mathrm{s}$ in $\mathrm{Cu}$ plasmas, stronger than that in $\mathrm{CH}$ 
$(\sim 300 \mathrm{~km} / \mathrm{s})$. This implies that the self-generated magnetic fields in $\mathrm{Cu}$ plasmas are much more concentrated and compressed in the cold dense surface region, providing a good explanation for the experimental observations in 2 (c).

In addition, the Righi-Leduc effect induced by plasma magnetization leads to bending of the heat flux $\mathbf{q}_{e}=$ $\left\langle\frac{1}{2} m_{e} v^{2} \mathbf{v}\right\rangle_{f 1}$ and the Nernst convection $\mathbf{v}_{N} \sim \mathbf{q}_{e} /\left[(1 / \gamma-1) p_{e}\right]$ towards the direction of $-\mathbf{B} \times \nabla T_{e}$, orthogonal to both magnetic fields and electron temperature gradient, which, to some extent, play roles in inhibiting transport of magnetic field towards the cold dense region. Figures $4(\mathrm{e})$ and $4(\mathrm{f})$ plot distributions of plasma magnetization parameter $\chi_{e} \equiv \omega_{c e} \tau_{e i} \propto B T_{e}^{1.5} / \bar{Z} n_{e}$ in respectively $\mathrm{CH}$ and $\mathrm{Cu}$ plasmas. We see that, despite with relatively lower temperature $T_{e}$ and weaker magnetic field $B, \mathrm{CH}$ plasmas have larger $\chi_{e}$ than $\mathrm{Cu}$ plasmas, because they have lower ionization charge states Z. Furthermore, for both cases, $\chi_{e}$ drops rapidly from $\gtrsim 1.0$ near the focal spot to $\sim 0.1$ within a few 100s of $\mu \mathrm{m}$ away. In order to evaluate how significance the Righi-Leduc effect is, we calculate the rotation angles between the Nernst velocity and the temperature gradient as $\theta_{R L} \equiv \arcsin \left(\frac{\mathbf{v}_{N} \times \nabla T_{e}}{\left|\mathbf{v}_{N}\right|\left|\nabla T_{e}\right|}\right)$, which are displayed in Figs. $4(\mathrm{~g})$ and $4(\mathrm{~h})$. We see for both cases $\theta_{R L}$ has almost the same magnitude, which decreases from $90^{\circ}$ near the focal spot to about $30^{\circ}$ several $100 \mu \mathrm{m}$ away. Considering the self-generated magnetic fields majorly distribute just at several $100 \mu \mathrm{m}$ up to millimeter away from laser focal spot (see Fig. 2), we conclude that the Righi-Leduc effect has very limited influence on magnetic field evolution and transport.

\section{DISCUSSION}

The first 3D synchronous proton radiography experiment has been carried out for probing self-generated magnetic fields in laser-produced HED plasmas on XG-III laser facility that has the unique capability of synchronizing three pulses in respectively femtosecond, picosecond and nanosecond duration scales. The spatially and temporally highlyresolved radiographys show clearly different magnetic field dynamics in low-Z $\mathrm{CH}$ and high- $\mathrm{Z} \mathrm{Cu}$ plasmas. Abundant 3D filamentary structures of magnetic fields grow up in $\mathrm{CH}$ hot coronal plasmas, while for $\mathrm{Cu}$, the fields are majorly compressed along the cold dense surface region whose internal structures are pretty vague. A cross-scale combination of RMHD, PIC and VFP simulations reveals that the Weibel instabilities contribute to growth of field filaments in $\mathrm{CH}$ plasmas, which are, however, heavily suppressed by the collision effect in $\mathrm{Cu}$ plasmas, and instead, in the latter the strong Nernst convection effects lead to field compressions.

Our results have fundamental implications for dynamics of magnetic fields in almost all HED relevant researches including astrophysics and ICF. The low- $\mathrm{Z} \mathrm{CH}$ and high- $\mathrm{Z} \mathrm{Cu}$ plasmas can be representative of the HED plasmas from respectively laser-ablated capsule (generally CD) in the direct-drive ICF scheme and laser-irradiated hohlraum wall (generally $\mathrm{Au}$ ) in the indirect-drive scheme. Furthermore, our 3D proton radiography methodology is applicable to a broad class of basic physics experiments where precise, time-resolved field measurements are a necessity.

\section{METHODS}

\section{Experimental facility and 3D proton radiography technology}

The three-dimensional (3D) synchronous proton radiography experiments presented in this work are carried out at XingGuang-III (XG-III $)^{26}$ laser facility at the Science and Technology on Plasma Physics Laboratory of Research Center of Laser Fusion, China Academy of Engineering Physics. The laser facility can generate femtosecond, picosecond, and nanosecond beams with three pulses, i.e., $800 \mathrm{~nm}$, $1053 \mathrm{~nm}$, and $527 \mathrm{~nm}$, respectively. To the best of our knowledge, XG-III is the first facility which produces three laser pulses with different pulse widths and wavelengths. An optical synchronization technique, combining super continuum generation and femtosecond optical parametric amplification, was developed to ensure three beams are from the same source to achieve precise synchronization. The femtosecond beam is a double chirped-pulse-amplification Ti: sapphire laser which applies cross-polarized wave generation to improve the temporal contrast. The picosecond/nanosecond beams utilize the optical parametric amplification + Nd: glass mixed amplification scheme. The output energy and pulse width of the three beams in our experiments are about $6 \mathrm{~J} / 50 \mathrm{fs}, 100 \mathrm{~J} / 1.5 \mathrm{ps}$, and $180 \mathrm{~J} / 2.0 \mathrm{~ns}$, respectively. The smallest synchronization time (peak-to-valley) and the shot-to-shot timing jitter (peak-to- peak) of less than $1.32 \mathrm{ps}$ have been achieved for the femtosecond and picosecond pulses.

The 3D synchronous proton radiography is set up based on the synchronized, orthogonal picosecond and femtosecond laser pulses, which can drive target normal sheath acceleration of protons of energies up to $10 \mathrm{MeV}$ from think $\mathrm{Cu}$ foils along both the face-on and side-on directions. These protons, generally having uniform distributions after propagation for mm distances, pass through HED plasmas from both face-on and side-on directions as well and subsequently deposit energy on the HD-V2 Radiochromic film (RCF) stacks. While inside the HED plasma, the radiography protons experience deflection by the Lorentz forces arising from magnetic fields, which result in a non-uniform dose-flux distributions on both RCF stacks. The response of the HD-V2 RCF stacks to proton dose has been well calibrated with a good linear relationship between proton dose and the measured optical density (OD). The resulting RCF images in both directions are scanned and analyzed to extract the 3D structures of self-generated magnetic fields by noting this linear relationship. 


\section{Path-integrated field reconstruction}

The extraction of the path-integrated magnetic field $\psi$ from proton radiographs in this work is done by using the algorithm and program PROBLEM ${ }^{28}$, where a parabolic Monge-Ampère equation is solved. Note that the formation of the proton radiographys can be understanding as following.

First, the propagation of laser-produced proton beam initially can be regarded as a uniform expansion from a point source (laser-driven target normal sheath acceleration region) into the probing HED plasma region. Under the paraxial approximation, $l_{p} / r_{i} \ll 1$, when the proton beam arrives at the probing region, it can be regarded as a two-dimensional near-planar sheet, where $l_{p}$ and $r_{i}$ are respectively the plasma lateral length and the distance from the proton source to the plasma, in our experiments, $l_{p} \approx 1 \mathrm{~mm}$ and $r_{i}=h \approx 10 \mathrm{~mm}$.

Afterwards, the proton beam is deflected by the Lorentz force arising from self-generated magnetic fields in HED plasmas, and the lateral deflection velocity can be expressed as

$$
\mathbf{v}\left(\mathbf{x}_{\perp 0}\right) \approx \frac{e}{m_{p} c} \hat{\mathbf{z}} \times \int_{0}^{l} l_{z} \mathrm{~d} s \mathbf{B}(\mathbf{x}(s))
$$

where $e$ is the proton charge, $m_{p}$ is the proton mass, $\mathbf{x}_{\perp 0}$ and $\mathbf{x}(s)$ are respectively the initial perpendicular position and the proton trajectory. The outgoing proton beam moves approximately in a straight line at a constant velocity, and the lateral deflection velocity $\mathbf{v}\left(\mathbf{x}_{\perp 0}\right)$ is amplified as

$$
\mathbf{x}_{\perp}^{(s)}\left(\mathbf{x}_{\perp 0}\right) \approx \frac{r_{i}+r_{s}}{r_{i}} \mathbf{x}_{\perp 0}+\frac{\mathbf{v}\left(\mathbf{x}_{\perp 0}\right)}{v_{p}} r_{s}
$$

where $v_{p}$ is the proton velocity, $r_{s}$ is the distance from the plasma to the RCF screen, $r_{s}=L \approx 50 \mathrm{~mm}$ in experiments, and $\mathbf{x}_{\perp}^{(s)}$ is the proton position on the screen.

Eventually, the deflection effect by the magnetic field makes the initially uniform proton beam redistribute on the RCF screen as

$$
\Psi\left(\mathbf{x}_{\perp}^{(s)}\left(\mathbf{x}_{\perp 0}\right)\right)=\frac{1}{\left|\operatorname{Det}\left(\nabla_{\perp 0}\left[\mathbf{x}_{\perp}^{(s)}\left(\mathbf{x}_{\perp 0}\right)\right]\right)\right|} \Psi_{0},
$$

where $\Psi$ and $\Psi_{0}$ are respectively the redistributed proton flux on the RCF screen and the initial proton flux (generally uniform), and $\nabla_{\perp 0}$ is the gradient operator of the initial plasma coordinates.

Therefore, the path-integrated magnetic field can be obtained by solving the Monge-Ampère equation (4), and

$$
\psi \equiv \int_{0}^{l_{z}} \mathrm{~d} s \mathbf{B}(\mathbf{x}(s))=-\frac{m_{p} c}{e} \hat{\mathbf{z}} \times \mathbf{v}\left(\mathbf{x}_{\perp 0}\right) .
$$

This inversion problem is well-defined, the geometric distance and proton energy are determined in the experiments, the redistributed proton flux $\Psi$ can be obtained from the response function of RCF optical density (OD) to proton dose, and which has an almost linear relationship in the low-dose region. The inhomogeneity of the initial proton flux $\Psi_{0}$ is ignored, see also Fig. S1 in Supplementary.

For the statistics of the revealed stochastic filamentary magnetic field in $\mathrm{CH}$ plasma, we perform the unidirectional Fourier transform to get the magnetic energy spectrum $E_{B}(k)$, and then take the statistical average. For example, for those in Fig. 2(f)-2(h), the main component of the magnetic field is $B_{z}$ and the wave number is mainly in the $x$ direction. So, we perform Fourier transform of the $B_{z}$ component in the $x$ direction, and then take statistical averaging in the $z$ direction to obtain the average spatial scale $\bar{\lambda}$.

\section{Radiation-magnetohydrodynamic (RMHD) simulation}

The RMHD simulations in this work are carried out by using the code "FLASH" ${ }^{29}$, which has been developed to include many high energy density physics modeling capabilities including laser energy deposition, multi-temperature $\left(T_{e} \neq T_{i} \neq T_{\text {rad }}\right)$, anisotropic electron thermal conductivity, and radiation transport etc. We have also further extended the code with the self-consistent magnetic field modeling capabilities including the Biermann battery, Nernst advection, resistive diffusion, Righi-Leduc and so on, that is the reason why we called it as "RMHD". The initial conditions of three-dimensional (3D) RMHD simulations are taken following those in the experiments. The size of the simulation box is $4 \times 4 \times 4 \mathrm{~mm}^{3}$ in all $(x, y, z)$ directions and the solid $\mathrm{CH} / \mathrm{Cu}$ foil targets with thicknesses $30 \mu \mathrm{m}$ in $\mathrm{z}$ direction and areas of $3.0 \times 0.8 \mathrm{~mm}^{2}$ in respectively $\mathrm{x}$ and $\mathrm{y}$ directions are placed at the position $z=1 \mathrm{~mm}$. the ns laser pulse with energy of $180 \mathrm{~J}$, flat-top temporal profile of duration $1.9 \mathrm{~ns}$ plus $0.1 \mathrm{~ns}$ rising and falling times, and spot diameter of about $160 \mu \mathrm{m}$ is incident on the foil targets at an oblique angle of about $45^{\circ}$. The equation of state (EOS) and opacity of solid foil materials $(\mathrm{CH}$ and $\mathrm{Cu})$ come from the code BADGER $^{38}$ and IONMIX ${ }^{39}$, respectively. The initial densities of the foils are set to $\rho_{\mathrm{CH}}=1.02 \mathrm{~g} / \mathrm{cm}^{3}$ and $\rho_{\mathrm{Cu}}=8.92 \mathrm{~g} / \mathrm{cm}^{3}$. All initial temperatures are set to be uniform as room temperatures $T_{0} \sim 290 \mathrm{~K}$.

\section{Collisional particle-in-cell (PIC) simulation}

The 2D3V PIC simulations in this work are carried out by using the fully-relativistic collisional PIC code "EPOCH" 30 . The simulation box is composed of $1536^{2}$ cells with the size $\left[-L_{x}, L_{x}\right] \times\left[-L_{y}, L_{y}\right]$, where $L_{x}=L_{y}=38.4 d_{i 0}$ and $d_{i 0} \equiv c / \omega_{p i 0}$ is the initial ion skin depth. Both profiles of the $\mathrm{CH} / \mathrm{Cu}$ foil electron densities $n_{e}$ and temperatures $T_{e}$ are set, according to the RMHD simulations (see Supplementary Fig. S2), as $n_{e}(r)=\left(n_{e 0}-n_{e b}\right) \exp \left(-r / L_{0}\right)+n_{e b}$ and $T_{e}(r)=2 T_{i}(r)=\left(T_{e 0}-T_{e b}\right) \exp \left(-r / L_{0}\right)+T_{e b}$, where $r=\sqrt{x^{2}+y^{2}}$, the gradient length is $L_{0}=6.4 d_{i 0}, n_{e 0}$ and $T_{e 0}$ are respectively the density and temperature of the coronal plasmas, $n_{e b}=0.01 n_{e 0}$ and $T_{e b}=0.1 T_{e 0}$ are taken for those as the background plasmas. In order to overcome the rarefaction caused by expansion and maintain the plasma profiles, we continuously inject isotropic thermal plasma in the central area where $r<10 d_{i 0}$ to mimic target ablation. All plasmas are initially set with isotropic Maxwellian distributions and in the simulations 64 macro-particles per cell for plasmas are employed. Thermal boundary conditions for particles and open boundary conditions for fields are taken. For the collisions, the binary collision algorithm ${ }^{40}$ is taken. All physical quantities are normalized as length to $d_{i 0}$, time to $L_{0} / v_{t e 0}$, and magnetic field to $\sqrt{2 \mu_{0} n_{e 0} T_{e 0}}$. 


\section{Vlasov-Fokker-Planck (VFP) simulation}

The 2D3P VFP simulations in this work are carried out by using the code "OSHUN"37, where the plasma distribution functions are expanded into an arbitrary number of spherical harmonics $f(r, p, t)=\sum_{l=0}^{\infty} \sum_{m=-l}^{l} f_{l}^{m}(r, p, t) P_{l}^{|m|}(\cos \theta) \exp (i m \varphi)$. In the $\operatorname{code}$, the collision operator, magnetic field generation and nonlocal heat transport, and also the implicit electric field solver and the laser inverse bremsstrahlung operator are naturally and self-consistently included. Due to the collision helps to achieve isotropization, we take $l=m=1$, which is enough to characterize the main physics. In order to reduce the computation resources of such VFP simulations down to a practical level, in the simulations the plasma profiles and the laser envelopes are adjusted within a reasonable range, in comparison with the RMHD results, where the details are shown in the Supplementary Fig. S6 and the corresponding text there. The plasma density profile is set as $\left.n_{e}(x, y)=2 \times 10^{22}[1.005-\tanh ((y-100 \mu m) / 50 \mu m))\right] \times\left[0.75 \exp \left(-(x / 300 \mu m)^{2}\right)+0.25\right] \mathrm{cm}^{-3}$, which makes $n_{e}$ transit smoothly from $4 \times 10^{22} \mathrm{~cm}^{-3}$ to $2.5 \times 10^{19} \mathrm{~cm}^{-3}$, and the simulation box is also large enough to avoid the boundary effects. The heating source profile is $I(x, y, t)=5.0 \times 10^{14}[0.5(\tanh ((y-130 \mu m) / 40 \mu m)+1.0)] \times[1.0+\cos (2 \pi x / 160 \mu m)] \times H(200 p s-t)$, where $H(t)$ is the Heavside function, this profile mimics the laser focal spot $160 \mu \mathrm{m}$ and average intensity $5.0 \times 10^{14} \mathrm{~W} / \mathrm{cm}^{2}$ in the experiments. We keep the laser heating for $200 \mathrm{ps}$, and then turn off the heat source to let the system continue to evolve freely for $100 \mathrm{ps}$. The average ion charges $\bar{Z}$ of $\mathrm{CH}$ and $\mathrm{Cu}$ plasma are set to be 3.5 and 25 , respectively, according to the ionization in the RMHD simulations, see Supplementary Fig. S2.

\section{REFERENCES}

${ }^{1}$ Kulsrud, R. M. \& Zweibel, E. G. On the origin of cosmic magnetic fields. Reports Prog. Phys. 71, 046901 (2008).

${ }^{2}$ Lebedev, S. V., Frank, A. \& Ryutov, D. D. Exploring astrophysics-relevant magnetohydrodynamics with pulsed-power laboratory facilities. Rev. Mod. Phys. 91, 025002 (2019).

${ }^{3}$ Gregori, G. et al. Generation of scaled protogalactic seed magnetic fields in laser-produced shock waves. Nature 481, 480-483 (2012).

${ }^{4}$ Kugland, N. L. et al. Self-organized electromagnetic field structures in laser-produced counter-streaming plasmas. Nat. Phys. 8, 809-812 (2012).

${ }^{5}$ Drake, R. P. High-Energy-Density Physics: Foundation of Inertial Fusion and Experimental Astrophysics. Springer, Second Edition (2018).

${ }^{6}$ Haines, M. G. Magnetic-field generation in laser fusion and hot-electron transport. Can. J. Phys. 64, 912-919 (1986).

${ }^{7}$ Biermann, L. Über den Ursprung der Magnetfelder auf Sternen und im interstellaren Raum. Z. Naturforsch. 5a, 65-71 (1950).

${ }^{8}$ Nishiguchi, A., Yabe, T. \& Haines, M. G. et al. Convective amplification of magnetic fields in laser-produced plasmas by the Nernst effect. Phys. Rev. Lett. 53, 262-265 (1984).

${ }^{9}$ Luciani, J. F., Mora, P. \& Bendib, A. Magnetic field and nonlocal transport in laser-created plasmas. Phys. Rev. Lett. 55, 2421-2424 (1985).

${ }^{10}$ Ridgers, C. P., Kingham, R. J. \& Thomas, A. G. R. Magnetic cavitation and the reemergence of nonlocal transport in laser plasmas. Phys. Rev. Lett. 100, 075003 (2008).

${ }^{11}$ Braginskii, S. I. Transport Properties in a Plasma, Review of Plasma Physics Vol. 1, edited by M. A. Leontovish (Consultants Bureau, New York, 1966), p. 205.

${ }^{12}$ Kho, T. H. \& Haines, M. G. Nonlinear Kinetic Transport of Electrons and Magnetic Field in Laser-Produced Plasmas. Phys. Rev. Lett. 55, 825-828 (1985).

${ }^{13}$ Manuel, M. J. E. et al. First measurements of Rayleigh-Taylor-induced magnetic fields in laser-produced plasmas. Phys. Rev. Lett. 108, 255006 (2012).

${ }^{14}$ Srinivasan, Bhuvana, Dimonte, Guy \& Tang, X.-Z. Magnetic field generation in Rayleigh-Taylor unstable inertial confinement fusion plasmas. Phys. Rev. Lett. 108, 165002 (2012).

${ }^{15}$ Weibel, E. S. Spontaneously Growing Transverse Waves in a Plasma Due to an Anisotropic Velocity Distribution. Phys. Rev. Lett. 2, 83-84 (1959).

${ }^{16}$ Davidson, R. C., Hammer, D. A., Haber, I. \& Wagner, C. E. Nonlinear development of electromagnetic instabilities in anisotropic plasmas. Phys. Fluids 15, 317-333 (1972).

${ }^{17}$ Huntington, C. M. et al. Observation of magnetic field generation via the Weibel instability in interpenetrating plasma flows. Nat. Phys. 11, $173-176(2015)$.

${ }^{18}$ Ruyer, C., Bolaños, S. \& Albertazzi, B. et al. Growth of concomitant laser-driven collisionless and resistive electron filamentation instabilities over large spatiotemporal scales. Nat. Phys. 16, 983-988 (2020).

${ }^{19}$ Nilson, P. M. et al. Magnetic reconnection and plasma dynamics in two-beam laser-solid interactions. Phys. Rev. Lett. 97, 255001 (2006).

${ }^{20} \mathrm{Li}, \mathrm{C}$. K. et al. Observation of megagauss-field topology changes due to magnetic reconnection in laser-produced plasmas. Phys. Rev. Lett. 99, 055001 (2007).

${ }^{21}$ Zhong, J. et al. Modelling loop-top X-ray source and reconnection outflows in solar flares with intense lasers. Nat. Phys. 6, 984-987 (2010).

${ }^{22}$ Rosenberg, M. J. et al. A laboratory study of asymmetric magnetic reconnection in strongly driven plasmas. Nat. Commun. 6, 6190 (2015).

${ }^{23}$ Tubman, E. R. et al. Observations of pressure anisotropy effects within semi-collisional magnetized plasma bubbles. Nat. Commun. 12, 334 (2021).

${ }^{24}$ Borghesi, M. et al. Proton imaging detection of transient electromagnetic fields in laser-plasma interactions (invited). Rev. Sci. Instrum. 74, 1688-1693 (2003).

${ }^{25}$ Rygg, J. R. et al. Proton Radiography of Inertial Fusion Implosions. Science 319, 1223-1225 (2008).

${ }^{26}$ Zhu, Q. et al. The Xingguang-III laser facility: Precise synchronization with femtosecond, picosecond and nanosecond beams. Laser Phys. Lett. 15, 015301 (2018).

${ }^{27} \mathrm{Li}$, C. K. et al. Measuring E and B fields in laser-produced plasmas with monoenergetic proton radiography. Phys. Rev. Lett. 97, 135003 (2006).

${ }^{28}$ Bott, A. F. A. et al. Proton imaging of stochastic magnetic fields. J. Plasma Phys. 83, 905830614 (2017)

${ }^{29}$ Fryxell, B. et al. FLASH : AN ADAPTIVE MESH HYDRODYNAMICS CODE FOR MODELING ASTROPHYSICAL THERMONUCLEAR FLASHES. The Astrophysical Journal Supplement Series 131, 273-334 (2000).

${ }^{30}$ Arber, T. D. et al. Contemporary particle-in-cell approach to laser-plasma modelling. Plasma. Phys. Controlled. Fusion. 57, 113001 (2015).

${ }^{31}$ Schoeffler, K. M. \& Silva, L. O. Effects of collisions on the generation and suppression of temperature anisotropies and the Weibel instability. Phys. Rev. Research 2, 033233 (2020).

${ }^{32}$ Schoeffler, K. M., Loureiro, N. F. \& Silva, L. O. Fully kinetic Biermann battery and associated generation of pressure anisotropy. Phys. Rev. E 97, 033204 (2018).

${ }^{33}$ Schoeffler, K. M. M. \& Silva, L. O. O. General kinetic solution for the Biermann battery with an associated pressure anisotropy generation. Plasma Phys. Control. Fusion 60, 014048 (2018).

${ }^{34}$ Schoeffler, K. M., Loureiro, N. F., Fonseca, R. A. \& Silva, L. O. Magnetic-field generation and amplification in an expanding plasma. Phys. Rev. Lett. 112, 175001 (2014).

${ }^{35}$ Wallace, J. M., Brackbill, J. U., Cranfill, C. W., Forslund, D. W. \& Mason, R. J. Collisional effects on the Weibel instability. Phys. Fluids 30, 1085 (1987). 
${ }^{36}$ Ruyer, C., Gremillet, L., Debayle, A. \& Bonnaud, G. Nonlinear dynamics of the ion Weibel-filamentation instability: An analytical model for the evolution of the plasma and spectral properties. Phys. Plasmas 22, 032102 (2015).

${ }^{37}$ Tzoufras, M., Bell, A. R., Norreys, P. A. \& Tsung, F. S. A Vlasov-Fokker-Planck code for high energy density physics. J. Comput. Phys. 230, 6475-6494 (2011).

${ }^{38}$ Heltemes, T. A. \& Moses, G. A. BADGER v1.0: A Fortran equation of state library. Comput. Phys. Commun. 183, 2629-2646 (2012).

${ }^{39}$ Macfarlane, J. J. IONMIX-A CODE FOR COMPUTING THE EQUATION OF STATE AND RADIATIVE PROPERTIES OF LTE AND NON-LTE Plasmas. Comput. Phys. Commun. 56, 259-278 (1989).

${ }^{40}$ Nanbu, K. \& Yonemura, S. Weighted Particles in Coulomb Collision Simulations Based on the Theory of a Cumulative Scattering Angle. J. Comput. Phys. 145, 639-654 (1998).

\section{ACKNOWLEDGEMENTS}

This work is supported by Science Challenge Project, No. TZ2018005; National Natural Science Foundation of China, Grant No. 11825502 and 11921006; the Strategic Priority Research Program of Chinese Academy of Sciences Grant No. XDA25050900; the National Key R \& D Program of China, Grant No. 2016YFA0401100; BQ acknowledges Supported from National Natural Science Funds for Distinguished Young Scholar, Grant No. 11825502. The simulations are carried out on the Tianhe-2 supercomputer at the National Supercomputer Center in Guangzhou.

\section{AUTHOR CONTRIBUTIONS}

B.Q. and S.P.Z. proposed and were in charge of the research campaign as principle investigators. Z.H.Z., Z.L.,Y.X., W.Q.Y. and B.Q. carried the simulations, the data analysis. S.K.H., H.H.A., Z.H.Z., Z.L., J.J.Ye, Z.Y.X., J.X., Z.H.F., W.W., W.M.Z. and B.Q. carried out the experiments. B.Q., Z.H.Z. and S.P.Z. wrote the paper. B.H.Z. and X.T.H. contribute to theoretical interpretations of the results.

\section{COMPETING INTERESTS}

The authors declare no competing interests.

\section{ADDITIONAL INFORMATION}

Correspondence and requests for materials should be addressed to B.Q. or S.P.Z. 


\section{Supplementary Files}

This is a list of supplementary files associated with this preprint. Click to download.

- SupplementaryInformation.pdf 